\title{
Anatomia da madeira de quatro espécies do gênero Miconia Ruiz \& Pavón (Melastomataceae)*
}

\author{
Micheline Leite Marcon ${ }^{12}$ \\ Cecília Gonçalves Costa ${ }^{2} 3$
}

\section{RESUMO}

Neste trabalho faz-se a descrição anatômica das madeiras de Miconia cabussu Hoehne, Miconia cinnamomifolia (DC.) Naudin, Miconia rigidiuscula Cogn. e Miconia sellowiana Naudin. As quatro espécies apresentam camadas de crescimento distintas; vasos solitários e em múltiplos; placas de perfuração simples; pontoações intervasculares pequenas, alternas e ornamentadas; parênquima axial paratraqueal escasso; raios integrados por células predominantemente quadradas e eretas; fibras semelhantes a parênquima, septadas e gelatinosas. As características que se mostraram úteis para a separação das espécies foram: demarcação das camadas de crescimento em M. cabussu e M. sellowiana; presença de traqueóides em $M$. cabussu; pontoações raio-vasculares em $M$. rigidiuscula e arranjo das fibras semelhantes a parênquima em M. cinnamomifolia. A forma das pontoações intervasculares e a largura dos raios separam as espécies em dois grupos: um inclui $M$. cabussu e $M$. cinnamomifolia e o outro, $M$. rigidiuscula e $M$. sellowiana. A formação dos grupos está representada em dendrogramas construídos através da análise de agrupamento.

Palavras-chaves: Anatomia da madeira, Miconia, Melastomataceae

\begin{abstract}
The wood anatomy of Miconia cabussu Hoehne, Miconia cinnamomifolia (DC.) Naudin, Miconia rigidiuscula Cogn. and Miconia sellowiana Naudin is described. The four species show distinct growth rings; solitary and multiples vessels; simple perforated plates; small, alternate and vestured intervessel pits; paratracheal scanty axial parenchyma; rays are composed by square, upright and few procumbent cells; parenchyma-like fibre; septate and gelatinous fibres. The useful characteristics for separation the species are: growth rings boundaries in $M$. cabussu and $M$. sellowiana; vascular tracheids present in M. cabussu; vessel-ray pits in M. rigidiuscula and parenchyma-like fibres arrangement in $M$. cinnamomifolia. The shape of the intervessel pits and the ray width separate the species into two groups: one includes M. cabussu and M. cinnamomifolia, the other group includes M. rigidiuscula and M. sellowiana. Dendrograms done in the cluster analysis represent these groups.
\end{abstract}

Keywords: wood anatomy, Miconia, Melastomataceae

\footnotetext{
*Parte da Dissertação de Mestrado da primeira autora

${ }^{1}$ Bolsista da Fundação Botânica Margaret Mee

${ }^{2}$ Instituto de Pesquisas Jardim Botânico do Rio de Janeiro

Rua Jardim Botânico, 1008 - Jardim Botânico

Rio de Janeiro - RJ.

CEP: 22460-000

${ }^{3}$ Bolsista do CNPq
} 


\section{INTRODUÇÃO}

As Melastomataceae constituem uma das famílias mais importantes da flora neotropical, com cerca de 5000 espécies subordinadas a 185 gêneros e 11 tribos (Renner 1993) e apresentam um importante interesse ornamental por suas flores vistosas ou pela folhagem rajada ou pintada (Wurdack 1962). Dividida por Cogniaux (1891) em três subfamílias - Melastomatoideae, Astronoideae e Memecyloideae, as Melastomataceae apresentam uma história taxonômica complicada, tendo sua divisão em subfamílias sido modificada por diversos autores (Van Vliet et al. 1981, Baas 1981, Dahlgren \& Thorne 1984, Johnson \& Briggs 1984, Keating 1984, Mentink \& Baas 1992, Renner 1993).

Miconia Ruiz \& Pavon, o maior gênero da família, está subordinado à subfamília Melastomatoideae e à tribo Miconieae, sendo representado no Brasil por cerca de 250 espécies muito ornamentais (Barroso et al. 1984; Martins et al. 1996). Muitas dessas espécies são próprias da Floresta Atlântica, desempenhando importante papel na manutenção da ave-fauna local, que utiliza os frutos de algumas dessas espécies em sua alimentação (Relatório PMA 1995). A madeira das espécies deste gênero pode ser utilizada para a obtenção de carvão e empregada em carpintaria e em pequenas construções (Record \& Mell 1924).

De acordo com Baumgratz (1980), o gênero Miconia apresenta uma série de dificuldades quanto à identificação de suas espécies, muitas das quais são fundamentadas em características consideradas duvidosas por estarem sujeitas a variações. Problemas de ordem taxonômica ocorrem também no que diz respeito ao posicionamento de determinadas espécies nas seções estabelecidas por Cogniaux (1887/1888).

Tanto para a família quanto para o gênero, os estudos sobre a anatomia da madeira são escassos, ressaltando-se os de Van Vliet (1981), Van Vliet et al. (1981) e os de Welle \& Koek-Noorman $(1978,1981)$.
Diante disto, propõe-se neste trabalho o estudo anatômico da madeira de Miconia cabussu, Miconia cinnamomifolia, Miconia rigidiuscula e Miconia sellowiana, a fim de contribuir para um melhor conhecimento do gênero Miconia, em especial na Floresta Atlântica. Este ecossistema, do qual fazem parte as três primeiras espécies, encontra-se altamente ameaçado e está reduzido a $6 \%$ de sua área original (Programa Mata Atlântica 1993, Guedes-Bruni \& Lima 1994). além disso, tem-se como objetivo verificar se as características anatômicas do lenho dessas 4 espécies permitem confirmar o posicionamento d Miconia cabussu e Miconia cinnamomifolia na seção Glossocentrum e de Miconia rigidiuscula e Miconia sellowiana, na seção Chaenanthera. Estes dados poderão contribuir, juntamente com estudos posteriores, para definir o posicionamento seccional de Miconia sellowiana, espécie que segundo Wurdack poderia ser incluída na seção Glossocentrum por ser morfologicamente muito semelhante às espécies desta seção e que foi excluída por Baumgratz (1984) da seção Chaenanthera, podendo segundo o autor vir a constituir, com outras espécies, uma seção à parte.

\section{MATERIAL E MÉTODOS}

As amostras de Miconia cabussu, Miconia rigidiuscula e Miconia sellowiana foram adquiridas nas xilotecas do Instituto de Pesquisas Jardim Botânico do Rio de Janeiro e do Instituto de Pesquisas Tecnológicas de São Paulo. As amostras de Miconia cinnamomifolia foram coletadas na Reserva Biológica de Poço das Antas, município de Silva Jardim, RJ. Todo o material analisado encontra-se registrado na xiloteca do Instituto de Pesquisas Jardim Botânico do Rio de Janeiro: Miconia cabussu (RBw1598, Laminário 1973); Miconia cinnamomifolia ( $\mathrm{RBw}$ 7436, 7437, 7438 e 7446, Laminário 1871, 1872, 1876 e 1883); Miconia rigidiuscula (RBw 2175, Laminário 1972); Miconia sellowiana (RBw 1703, Laminário 
1971).

O material botânico foi processado no Laboratório de Botânica Estrutural do Instituto de Pesquisas Jardim Botânico do Rio de Janeiro, onde foram confeccionados os corpos de prova, posteriormente seccionados em micrótomo de deslizamento Jung $\mathrm{K}$, nos planos transversal e longitudinal (radial e tangencial). Após clarificação, desidratação e coloração, montaram-se as lâminas permanentes (Johansen 1940; Sass 1958; Burger \& Richter 1991) que foram integradas ao acervo da xiloteca do Instituto de Pesquisas Jardim Botânico do Rio de Janeiro. As aferições dos valores dimensionais das fibras e do comprimento dos elementos vasculares foram executadas em material dissociado (Jane 1956; Fedalto 1982) e macerado (Dop \& Gautié 1909). As descrições, mensurações e contagens dos elementos celulares obedeceram às normas do IAWA Committee (1989). Nas mensurações utilizou-se o Software Image ProPlus IPP WIM, acoplado ao microscópio binocular Olympus BH-2. Os valores mínimos, médios e máximos para cada tipo de medida e contagem realizadas encontram-se registrados nas descrições de cada espécie. A classificação das células em relação ao tamanho foi feita seguindo as recomendações da COPANT (1973). As fotomicrografias foram realizadas em microscópio Olympus $\mathrm{BH}-2$.

A fim de comparar e reunir em grupos as espécies estudadas, foi efetuada uma análise de agrupamento utilizando-se todas as características observadas. Esta análise foi realizada baseando-se numa matriz de distância de Manhattan para dados binários pelo método de peso proporcional (Weighted pair-group average) através do programa Statistica. Este método é aconselhado pela literatura (Davis 1973 apud Gonzalez-Rodrigues et al. 1989) por respeitar de maneira satisfatória a matriz original de correlação.

\section{RESULTADOS}

\section{DESCRIÇÕES ANATÔMICAS}

Miconia cabussu Hoehne (Figs. 1-7).

Camadas de crescimento: distintas, demarcadas pelo achatamento e maior espessamento parietal das fibras e pelo maior número de vasos no lenho tardio e, ainda, por porosidade em semi-anel (Fig. 1).

Elementos de vaso: porosidade em semi-anel (Fig. 1); (6-)10(-14) vasos $/ \mathrm{mm}^{2}$ no lenho inicial e (10-) $14(-18)$ vasos $/ \mathrm{mm}^{2}$ no lenho tardio; solitários (Fig. 1), em múltiplos de 2-4 elementos (Fig. 1) e raramente em cachos de 3-6 elementos; arranjo difuso; seção circular a oval; diâmetro tangencial de (82-)101 $(-129) \mu \mathrm{m}$ no lenho inicial e de (39-)60 (-80) $\mu \mathrm{m}$ no lenho tardio; comprimento de (179-)418(-671) $\mu \mathrm{m}$; paredes com $2 \mu \mathrm{m}$ de espessura em média; placas de perfuração simples nas paredes terminais (Fig. 2) e lateral (Fig. 3) dos vasos; presença de traqueóides vasculares (Fig. 3); pontoações intervasculares pequenas, alternas, circulares e ornamentadas (Fig. 4); pontoações raio-vasculares e parênquimo-vasculares com aréola reduzida a aparentemente simples, às vezes alongadas nos sentidos horizontal, vertical e diagonal.

Fibras libriformes: comprimento de (505-) 657(-934) $\mu \mathrm{m}$; diâmetro tangencial de (13-)16 (-21) $\mu \mathrm{m}$; lúmen de (5-)7(-12) $\mu \mathrm{m}$; paredes muito espessas; presença de fibras semelhantes a parênquima em pequenas áreas (Fig. 1) ou em faixas muito irregulares, alternadas com fibras típicas. Essas fibras diferenciam-se por apresentar paredes mais delgadas, lúmen mais largo (11-)14(-22) $\mu \mathrm{m}$ e alguns espaços intercelulares observados em seção transversal; as pontoações ocorrem na parede radial dos dois tipos de fibras; fibras gelatinosas e septadas presentes.

Parênquima axial: paratraqueal escasso, em séries de 2 a 9 células, com (250-)362 $(-517) \mu \mathrm{m}$ de altura.

Raios: (12-)15(-19)/mm; unisseriados (Fig. 5) e unisseriados com porções bisseriadas, integrados predominantemente por células quadradas e eretas, com raras procumbentes 


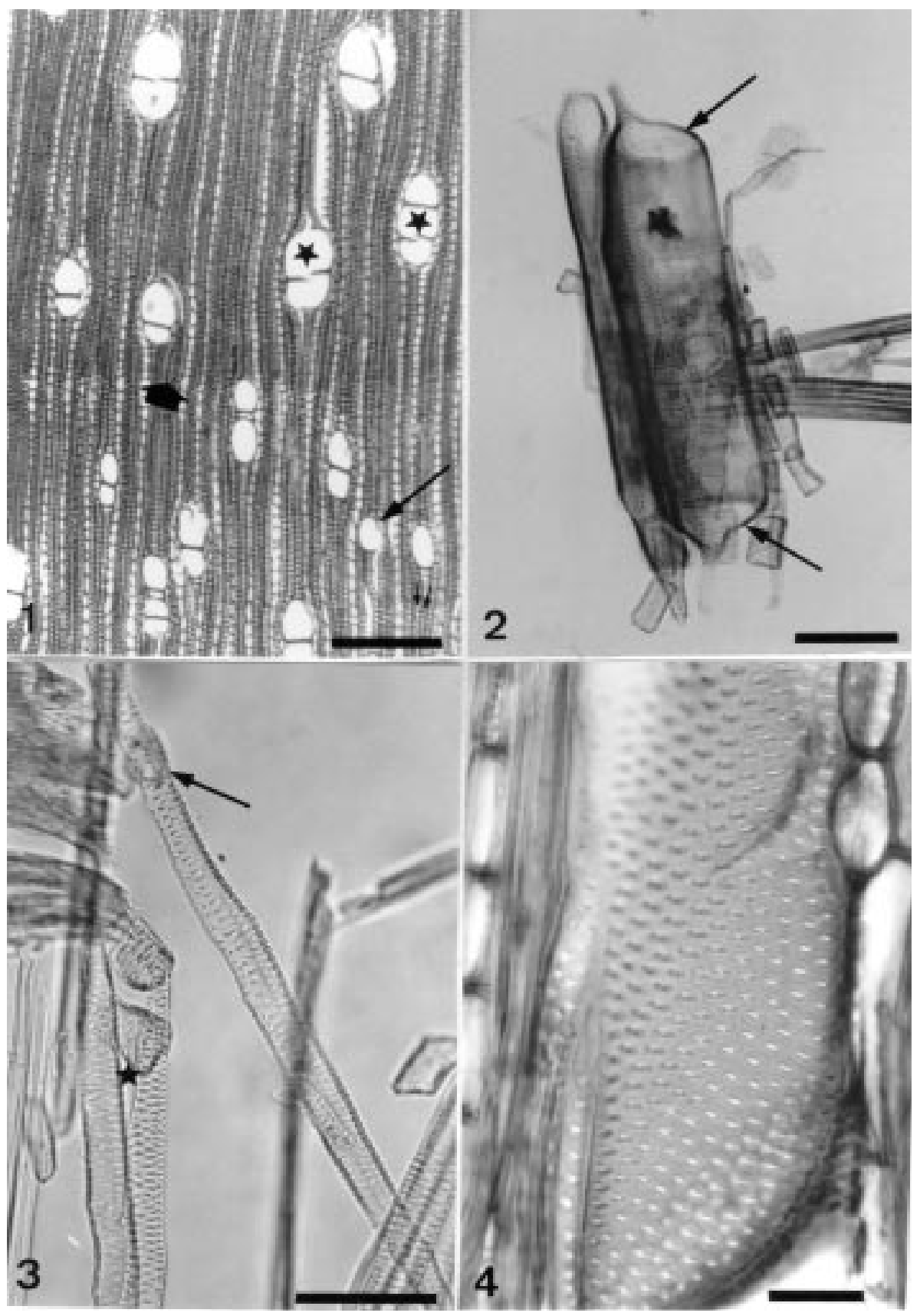

Figuras 1-4 - Miconia cabussu. Fig. 1: Seção transversal: Camada de crescimento demarcada pelo achatamento e maior espessamento parietal das fibras no lenho tardio $(\Rightarrow)$ e por uma diferença gradual no diâmetro dos elementos de vaso (porosidade em semi - anel); elementos de vaso solitários $(\rightarrow)$ e em múltiplos de 2 e 3 elementos ( $\star$ ); notar a presença de fibras semelhantes a parênquima com coloração mais clara em pequenas áreas $(\rightarrow)$; Barra $=300 \mu m$. Fig. 2: Elemento de vaso dissociado, em que se observam placas de perfuração simples nas paredes terminais $(\rightarrow)$; Barra $=100 \mu \mathrm{m}$. Fig. 3: Elemento de vaso com placa de perfuração lateral $(\rightarrow)$ e traqueóides vasculares $(\star)$ observados em material dissociado; Barra $=75 \mu \mathrm{m}$. Fig. 4: Seção tangencial: Pormenor de um elemento de vaso em que se observa a forma e a disposição das pontoações intervasculares; Barra $=25 \mu \mathrm{m}$. 
(Fig. 6); largura de (14-)19(-29) $\mu \mathrm{m}$; altura de (172-)326(-578); raios fusionados presentes (Fig. 5).

Mácula medular: presente (Fig. 7).

Miconia cinnamomifolia (DC.) Naudin (Figs. 8-12; 14).

Camadas de crescimento: distintas, demarcadas pelo achatamento e maior espessamento parietal das fibras no lenho tardio (Fig. 8).

Elementos de vaso: porosidade difusa (Fig. 8); (3-)7(-12) vasos/ $\mathrm{mm}^{2}$; solitários (Fig. 8), em múltiplos de 2-4 elementos (Fig. 8) ou raramente em cachos de 3-4 elementos; arranjo difuso; seção circular a oval; diâmetro tangencial de (81-)147(-216) $\mu \mathrm{m}$; comprimento de (320-)572(-899) $\mu \mathrm{m}$; paredes com $4 \mu \mathrm{m}$ de espessura, em média; placas de perfuração simples; pontoações intervasculares pequenas, alternas, circulares e ornamentadas (Fig. 9); pontoações raio-vasculares e parênquimovasculares semelhantes em forma e tamanho às intervasculares (fig. 10), algumas alongadas nos sentidos horizontal e vertical.

Fibras libriformes: comprimento de (627-) 875(-1474) $\mu \mathrm{m}$; diâmetro tangencial de (10-)19(-24) $\mu \mathrm{m}$; lúmen de (4-)10(-17) $\mu \mathrm{m}$; paredes delgadas a espessas; presença de faixas contínuas de fibras semelhantes a parênquima alternadas com fibras típicas (Fig. 11). As fibras que compõem essas faixas diferenciam-se por apresentar paredes um pouco mais delgadas, lúmen mais largo (13-) 20(-32) $\mu \mathrm{m}$ e muitos espaços intercelulares, observados em seção transversal (Fig. 12); as pontoações ocorrem na parede radial dos dois tipos de fibras; fibras gelatinosas (Fig. 12) e septadas presentes.

Parênquima axial: paratraqueal escasso (Fig. 11), em séries de 2 a 14 células (Fig. 14), com (151-)511(-693) $\mu \mathrm{m}$ de altura.

Raios: (10-)14(-17)/mm; unisseriados e unisseriados com porções bisseriadas (Fig. 14), integrados predominantemente por células quadradas e eretas, com raras procumbentes; largura de (13-)21(-36) $\mu \mathrm{m}$; altura de (124-)
383(-976) $\mu \mathrm{m}$; raios fusionados presentes. Mácula medular: presente.

Miconia rigidiuscula Cogn. (Figs. 13; 15-16). Camadas de crescimento: pouco distintas, demarcadas pelo achatamento e maior espessamento parietal das fibras no lenho tardio.

Elementos de vaso: porosidade difusa; (14-)17(-23) vasos $/ \mathrm{mm}^{2}$; solitários, em múltiplos de 2-4 elementos e em cachos de 4 e às vezes 6 elementos; arranjo difuso; seção circular a oval; diâmetro tangencial de (38-)72(-104) $\mu \mathrm{m}$; comprimento de (273-)399(-529) $\mu \mathrm{m}$; paredes com $2 \mu \mathrm{m}$ de espessura em média; placas de perfuração simples; pontoações intervasculares pequenas, alternas, poligonais e ornamentadas (Fig. 13); pontoações raiovasculares e parênquimo-vasculares com aréola reduzida a aparentemente simples, às vezes alongadas nos sentidos horizontal e vertical. Ocorrem também pontoações raiovasculares de dois diferentes tipos (aparentemente simples e circulares com aréola distinta) e tamanhos em uma mesma célula do raio (Fig. 15).

Fibras libriformes: comprimento de (381-) 514(-649) $\mu \mathrm{m}$; diâmetro tangencial de (13-)17 (-22) $\mu \mathrm{m}$; lúmen de (6-)10(-14) $\mu \mathrm{m}$; paredes delgadas a espessas; presença de fibras semelhantes a parênquima em pequenas áreas ou em faixas irregulares, alternadas com fibras típicas. Essas fibras diferenciam-se por apresentar o lúmen mais largo (14-)18(-25) $\mu \mathrm{m}$ e alguns espaços intercelulares, observados em seção transversal; as pontoações ocorrem na parede radial dos dois tipos de fibras; fibras gelatinosas e septadas presentes.

Parênquima axial: paratraqueal escasso, em séries de 2 a 5 células, com (210-)305(467) $\mu \mathrm{m}$ de altura.

Raios: (13-)16(-20) /mm; uni, bi e trissseriados (Fig. 16), integrados predominantemente por células quadradas e eretas, com raras procumbentes; largura de (24-)32(-39) $\mu \mathrm{m}$; altura de (159-)305(-554) $\mu \mathrm{m}$; 


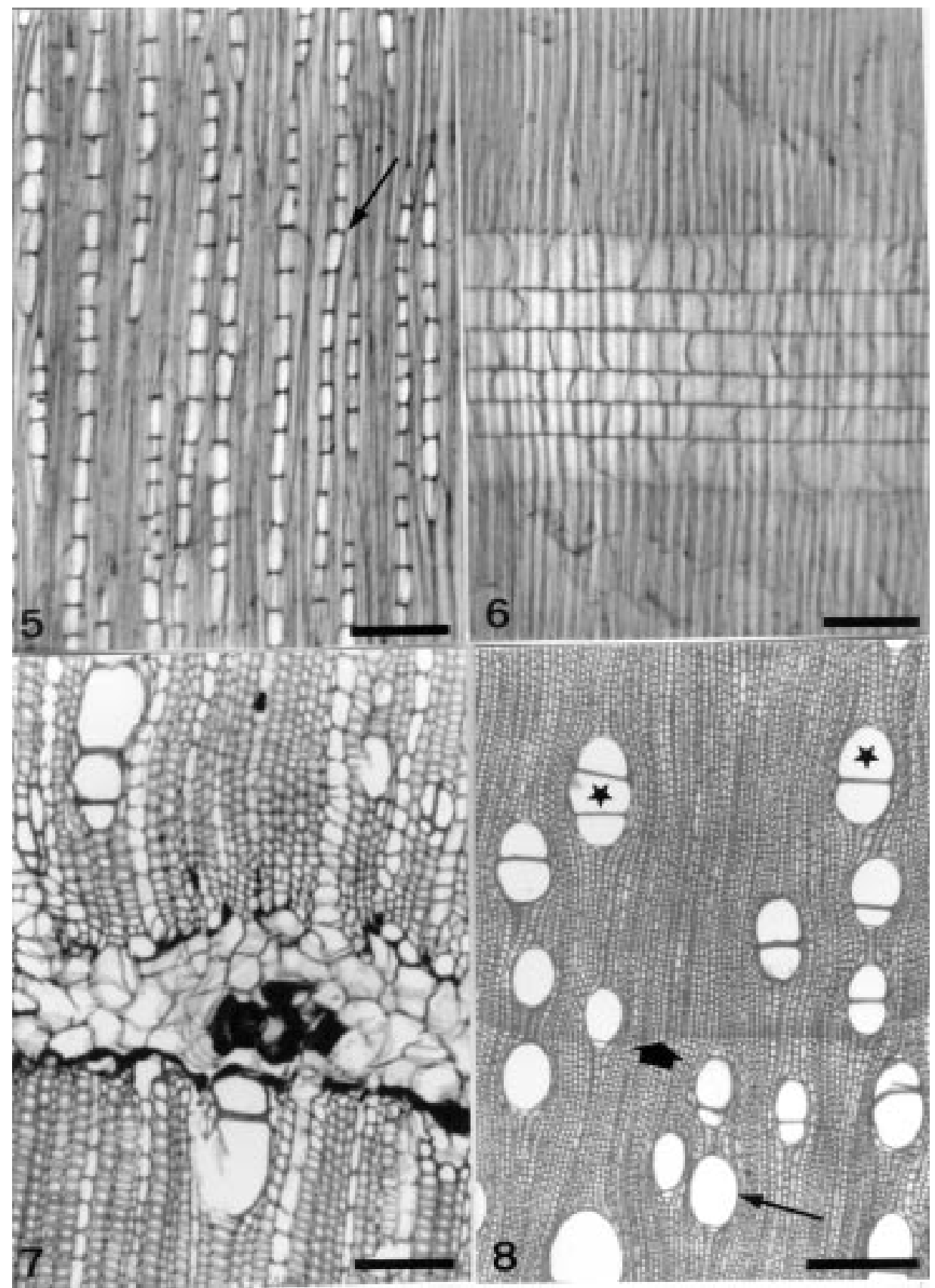

Figuras 5-7 - Miconia cabussu. Fig. 5: Seção tangencial, evidenciando raios unisseriados; nota-se a presença de raio fusionado $(\rightarrow)$; Barra $=100 \mu \mathrm{m}$. Fig. 6: Seção radial: raios integrados por células quadradas e eretas; Barra $=100 \mu \mathrm{m}$. Fig. 7: Seção transversal, evidenciando mácula medular; Barra $=100 \mu \mathrm{m}$. Figura 8 - Miconia cinnamomifolia . Seção transversal evidenciando camada de crescimento demarcada pelo achatamento e maior espessamento parietal das fibras no lenho tardio $(\Rightarrow)$; porosidade difusa; elementos de vaso solitários $(\rightarrow)$ e em múltiplos de 2 e 3 elementos $(\star)$; Barra $=300 \mu \mathrm{m}$. 

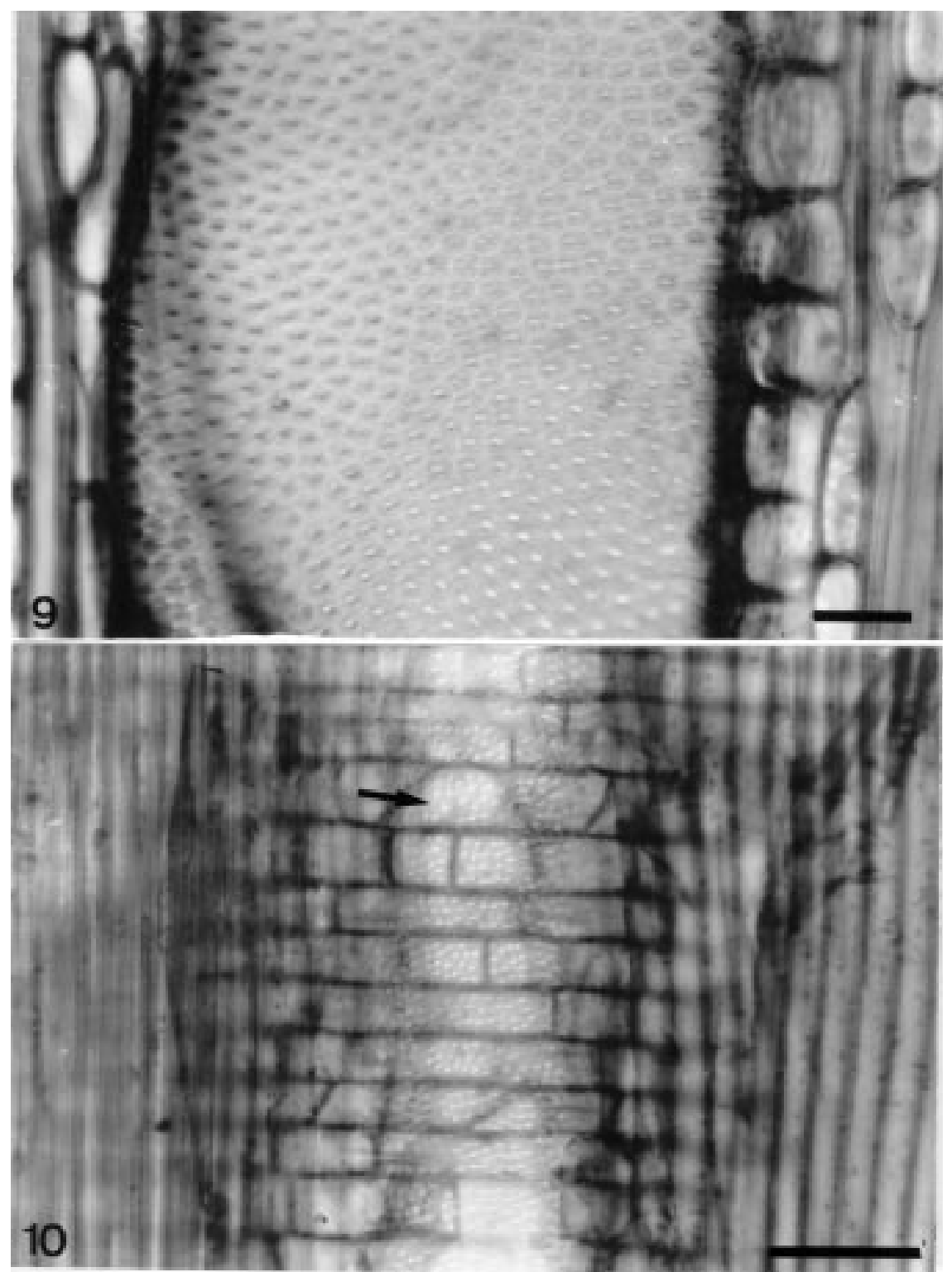

Figuras 9-10 - Miconia cinnamomifolia. Fig. 9: Seção tangencial: Pormenor de um elemento de vaso, evidenciando a forma e a disposição das pontoações intervasculares. Barra $=25 \mu \mathrm{m}$. Fig. 10: Seção radial: Detalhe das pontoações raio-vasculares. Barra $=75 \mu \mathrm{m}$.

raios fusionados presentes.

Mácula medular: presente.

Miconia sellowiana Naudin (Figs. 17-19).

Camadas de crescimento: distintas, demarcadas pelo achatamento e maior espessamento parietal das fibras e pelo maior número de vasos em arranjo tangencial no lenho tardio e, ainda, por uma faixa de fibras que se assemelham a parênquima (Fig. 17).
Elementos de vaso: porosidade difusa (Fig. 17); (27-)31(-36) vasos $/ \mathrm{mm}^{2}$ no lenho inicial e (40-)45(-50) vasos $/ \mathrm{mm}^{2}$ no lenho tardio; solitários, em múltiplos de 2-4 elementos e raramente em cachos de 3 elementos; arranjo difuso, alguns em arranjo tangencial no limite de demarcação do anel de crescimento (Fig. 17); seção circular a oval; diâmetro tangencial de (50-)63(-85) $\mu \mathrm{m}$; comprimento de (202-) $373(-537) \mu \mathrm{m}$; paredes com $4 \mu \mathrm{m}$ de espessura 

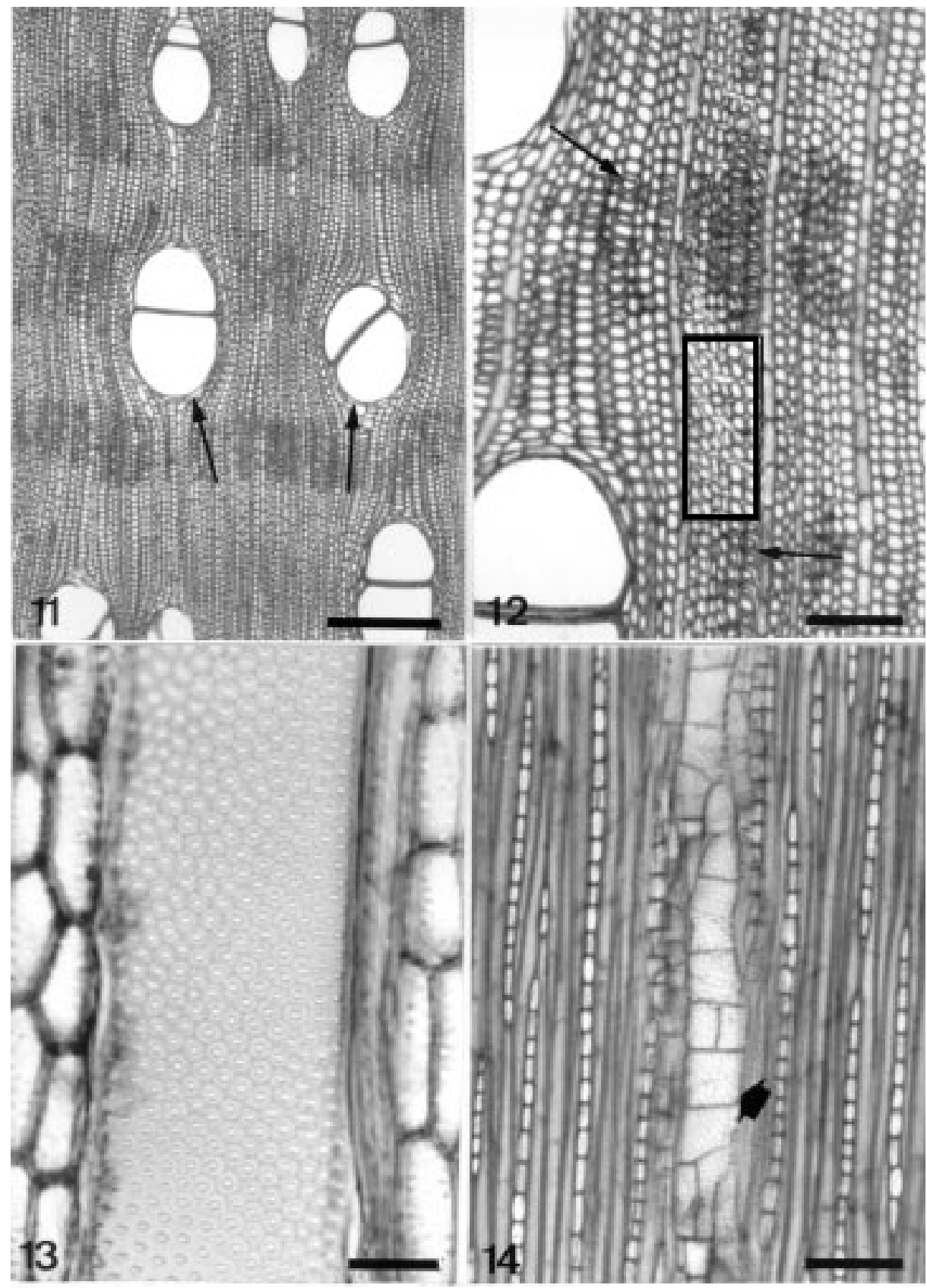

Figuras 11-12 - Miconia cinnamomifolia. Fig. 11: Seção transversal, evidenciando faixas de fibras típicas, de coloração mais escura alternadas com faixas de fibras semelhantes a parênquima que apresentam coloração mais clara; parênquima axial paratraqueal escasso $(\rightarrow)$. Barra $=300 \mu \mathrm{m}$. Fig. 12: Seção transversal: Detalhe das fibras semelhantes a parênquima $(\square)$ que se diferenciam por apresentar paredes mais delgadas, lúmen mais largo e espaços intercelulares; presença de fibras gelatinosas $(\rightarrow)$; Barra $=100 \mu \mathrm{m}$. Figura 13 - Miconia rigidiuscula - Seção tangencial. Pormenor de um elemento de vaso, evidenciando a forma e a disposição das pontoações intervasculares. Barra $=25 \mu \mathrm{m}$. Figura 14 - Miconia cinnamomifolia - Seção tangencial, evidenciando raios unisseriados e uma série de parênquima axial $(\overrightarrow{)})$. Barra $=100 \mu \mathrm{m}$. 


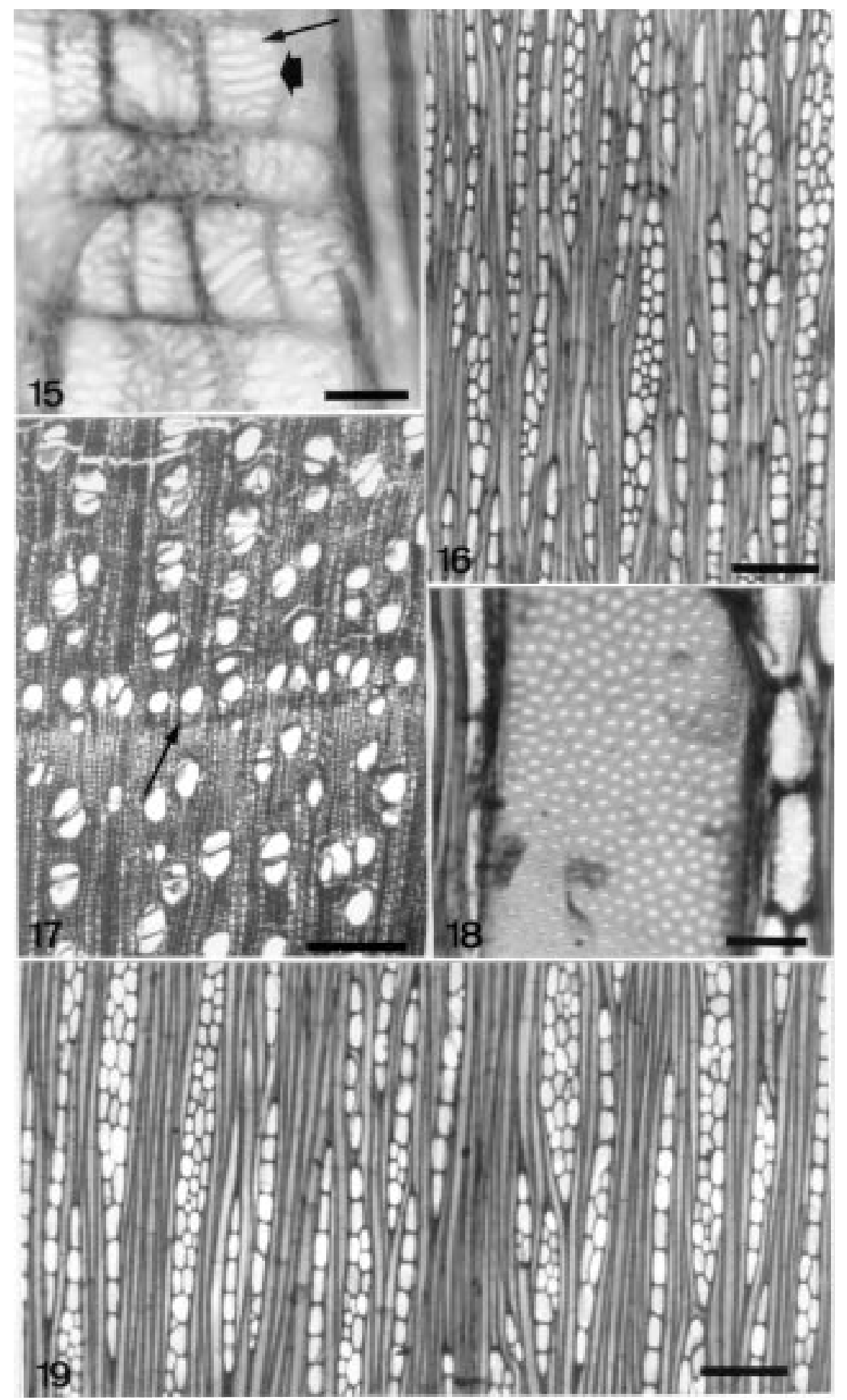

Figuras 15-16 - Miconia rigidiuscula - Fig. 15: Seção radial, evidenciando dois tipos de pontoações raio-vasculares em uma mesma célula do raio: aparentemente simples, algumas alongadas $(\rightarrow)$ e areoladas $(\rightarrow)$. Barra $=25 \mu m$. Fig. 16: Seção tangencial, em que se observam raios uni, bi e trisseriados. Barra $=100 \mu \mathrm{m}$. Figuras 17-19 - Miconia sellowiana - Fig. 17: Seção transversal: Camada de crescimento demarcada pelo achatamento e maior espessamento parietal das fibras e pelo maior número de vasos em arranjo tangencial no lenho tardio $(\rightarrow)$; porosidade difusa; nota-se no limite de demarcação da camada de crescimento a presença de uma faixa contínua de fibras com coloração mais clara, que se assemelha a uma faixa de parênquima marginal. Barra $=300 \mu \mathrm{m}$. Fig. 18: Seção tangencial: Pormenor de um elemento de vaso, evidenciando a forma e a disposição das pontoações intervasculares. Barra $=25 \mu \mathrm{m}$. Fig. 19: Seção tangencial, evidenciando raios uni, bi e trisseriados. Barra $=100 \mu \mathrm{m}$. 
em média; placas de perfuração simples; pontoações intervasculares pequenas, alternas, poligonais e ornamentadas (Fig. 18); pontoações raio-vasculares e parênquimovasculares semelhantes apenas em tamanho às intervasculares e de forma circular, com algumas alongadas nos sentidos horizontal e vertical.

Fibras libriformes: comprimento de (409-)621(-781) $\mu \mathrm{m}$; diâmetro tangencial de (12-)17 (-23) $\mu \mathrm{m}$; lúmen de (5-)8(-13) $\mu \mathrm{m}$; paredes espessas; presença de fibras semelhantes a parênquima em pequenas áreas ou em faixa contínua que se assemelha a um parênquima marginal no limite de demarcação do anel de crescimento (Fig. 17). Essas fibras diferenciam-se por apresentar paredes mais delgadas, lúmen mais largo (12-)17(-23) $\mu \mathrm{m}$ e poucos espaços intercelulares, observados em seção transversal; as pontoações ocorrem na parede radial dos dois tipos de fibras; fibras gelatinosas e septadas presentes.

Parênquima axial: paratraqueal escasso, em série de 4 a 9 células, com (225-)377 $(-558) \mu \mathrm{m}$ de altura.
Raios: (11-)14(-16)/mm; uni, bi e trisseriados (Fig. 19), integrados predominantemente por células quadradas e eretas, com raras procumbentes; largura de (25-)32(-39) $\mu \mathrm{m}$; altura de (167-)314(-557) $\mu \mathrm{m}$; raios fusionados presentes.

\section{ANÁLISE COMPARATIVA}

Analisando as diferenças qualitativas e quantitativas da madeira das quatro espécies estudadas, observa-se que alguns caracteres são exclusivos de cada espécie enquanto outros separam Miconia cabussu e $M$. cinnamomifolia de $M$. rigidiuscula e $M$. sellowiana (Tab. 1).

A indicação de que esta separação pode estar evidenciando a formação de dois grupos diferentes foi confirmada pela análise de agrupamento na qual obteve-se um dendrograma (Fig. 20) que mostra uma tendência de agrupamento entre Miconia cabussu e $M$. cinnamomifolia e entre $M$. rigidiuscula e $M$. sellowiana.

Tabela 1. Diferenças qualitativas e quantitativas da madeira das quatro espécies estudadas

\begin{tabular}{|c|c|c|c|c|}
\hline $\begin{array}{c}\text { CARACTERES/ } \\
\text { ESPÉCIES } \\
\end{array}$ & $\begin{array}{l}\text { Miconia } \\
\text { cabussu }\end{array}$ & $\begin{array}{c}\text { Miconia } \\
\text { cinnamomifolia }\end{array}$ & $\begin{array}{l}\text { Miconia } \\
\text { rigidiuscula }\end{array}$ & $\begin{array}{c}\text { Miconia } \\
\text { sellowiana } \\
\end{array}$ \\
\hline $\begin{array}{l}\text { Cam. cresc. demarcadas pelo a chatamento e espessamento das fibras no } \\
\text { lenho tardio }\end{array}$ & & $\mathrm{X}$ & $\mathrm{X}$ & \\
\hline $\begin{array}{l}\text { Cam. cresc. demarcadas pelo achatamento e espessamento das fibras, } \\
\text { maior número de vasos no L.T. e faixa de fibras semelhantes a } \\
\text { parênquima }\end{array}$ & & & & $\mathrm{X}$ \\
\hline $\begin{array}{l}\text { Cam. cresc. demarcadas pelo achatamento e espessamento das fibras, } \\
\text { maior número de vasos no L.T. e diferença gradual no diâmetro dos vasos }\end{array}$ & $\mathrm{X}$ & & & \\
\hline $\begin{array}{l}\text { Vasos } / \mathrm{mm}^{2} \\
\text { Diâmetro dos vasos }\end{array}$ & $\begin{array}{l}\text { PN } \\
\text { médios }\end{array}$ & $\begin{array}{l}\text { PN } \\
\text { médios }\end{array}$ & $\begin{array}{c}\mathrm{N} \\
\text { pequenos }\end{array}$ & $\begin{array}{c}\text { MN } \\
\text { pequenos }\end{array}$ \\
\hline Comprimento dos vasos & curtos & longos & curtos & curtos \\
\hline Pontoações intervasculares poligonais & & & $\mathrm{X}$ & $\mathrm{X}$ \\
\hline Pontoações intervasculares circulares & $\mathrm{X}$ & $\mathrm{X}$ & & \\
\hline $\begin{array}{l}\text { Pontoações raio-vasculares areoladas circulares } \\
\text { Pontoações raio-vasculares semelhantes às intervasculares quanto à forma } \\
\text { e tamanho }\end{array}$ & & $\mathrm{X}$ & $\mathrm{X}$ & $\begin{array}{l}\mathrm{X} \\
\mathrm{X}\end{array}$ \\
\hline Pontoações raio-vasculares com aréola reduzida a aparentemente simples & $\mathrm{X}$ & & $\mathrm{X}$ & \\
\hline $\begin{array}{l}\text { Pontoações raio-vasculares de dois tipos e tamanhos em uma mesma } \\
\text { célula }\end{array}$ & & & $\mathrm{X}$ & \\
\hline Traqueóides & $\mathrm{X}$ & & & \\
\hline Raios unisseriados ou unisseriados com porções bisseriadas & $\mathrm{X}$ & $\mathrm{X}$ & & \\
\hline $\begin{array}{l}\text { Raios uni, bi e trisseriados } \\
\text { Largura dos raios }\end{array}$ & MF & MF & $\begin{array}{l}\mathrm{X} \\
\mathrm{F}\end{array}$ & $\begin{array}{l}\mathrm{X} \\
\mathrm{F}\end{array}$ \\
\hline Fibras semelhantes a parênquima & PA e FI & FTC & PA e FI & PA e FCPM \\
\hline
\end{tabular}

Legenda: $(\mathrm{x})=$ presente; ()$=$ ausente; PN-pouco numerosos; N-numerosos; MN-muito numerosos; F-finos; MF-muito finos; PA-em pequenas áreas; FI-em faixas irregulares; FTC-em faixas tangenciais contínuas; FCPM-em faixa contínua que se assemelha a uma parênquima marginal. Cam. cresc. = camadas de crescimento. 
Comparando-se o lenho das quatro espécies estudadas com o de duas espécies da seção Glossocentrum descritas na literatura (Tab. 2), observa-se que alguns caracteres separam Miconia cabussu, M. cinnamomifolia, M. candolleana e $M$. minutiflora de $M$. rigidiuscula e $M$. sellowiana.
Esta separação foi confirmada através da análise de agrupamento. $\mathrm{O}$ dendrograma obtido (Fig. 21) evidencia a formação de um primeiro grupo principal formado por $M$. rigidiuscula e M. sellowiana. As demais espécies não formaram um grupo único, mas se posicionaram a uma distância que as separa daquele primeiro grupo formado.

Tabela 2. Tabela comparativa entre as espécies estudadas e as duas espécies descritas na literatura (Barbosa et al. 1978 e Welle \& Koek-Noorman 1981).

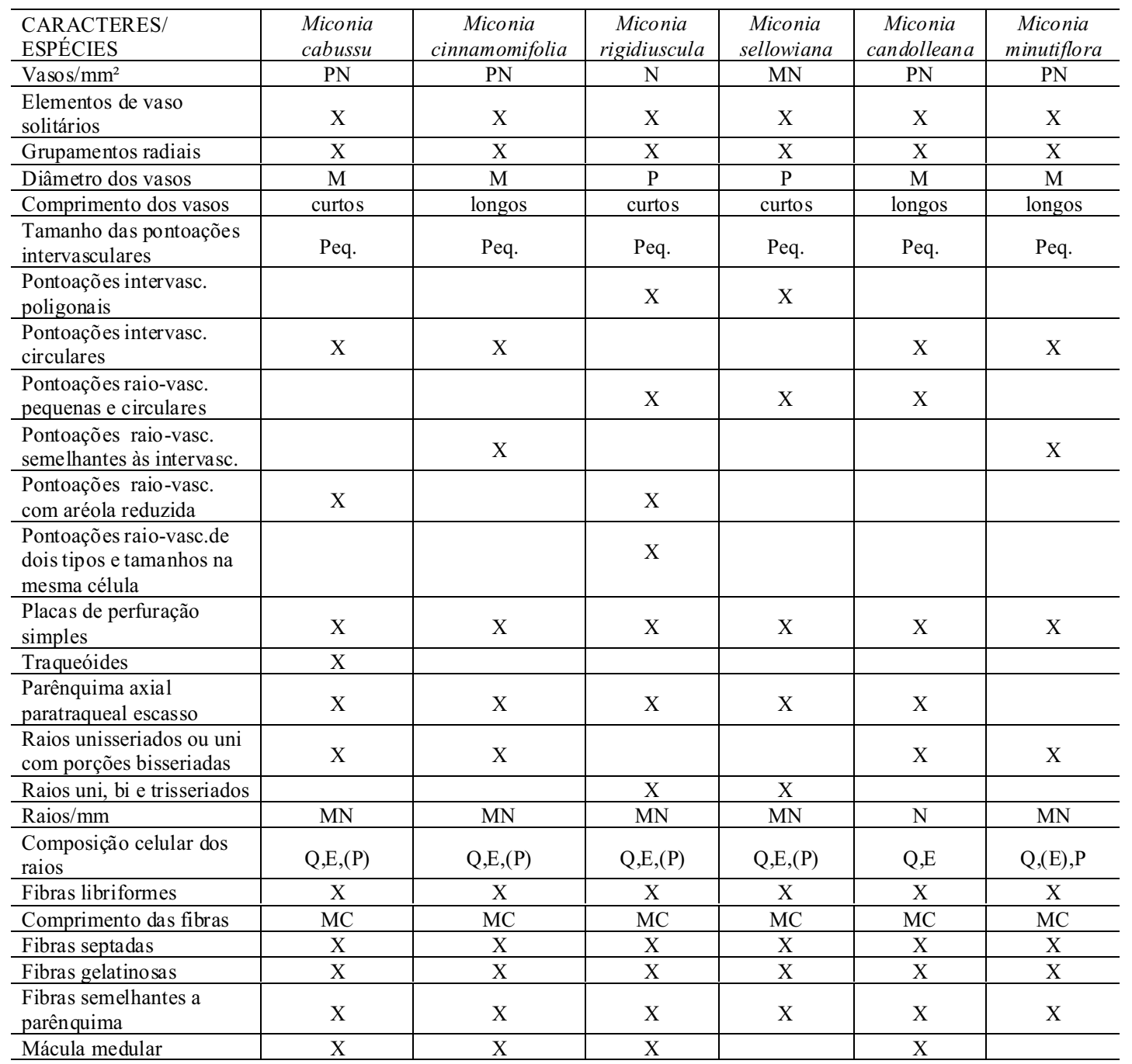

Legenda: $(X)=$ presente; ()$=$ ausente; Intervasc. = intervasculares; Cél. = célula; $P N=$ pouco numerosos; $N=$ numerosos; $\mathrm{MN}=$ muito numerosos; $\mathrm{M}=$ médio; $\mathrm{P}=$ pequeno; Peq. = pequena; $\mathrm{Q}=$ quadradas; $\mathrm{E}=\operatorname{eretas} ; \mathrm{P}=$ procumbentes; $(\mathrm{E})=$ poucas eretas; $(\mathrm{P})=$ poucas procumbentes; $\mathrm{MC}=$ muito curtas 


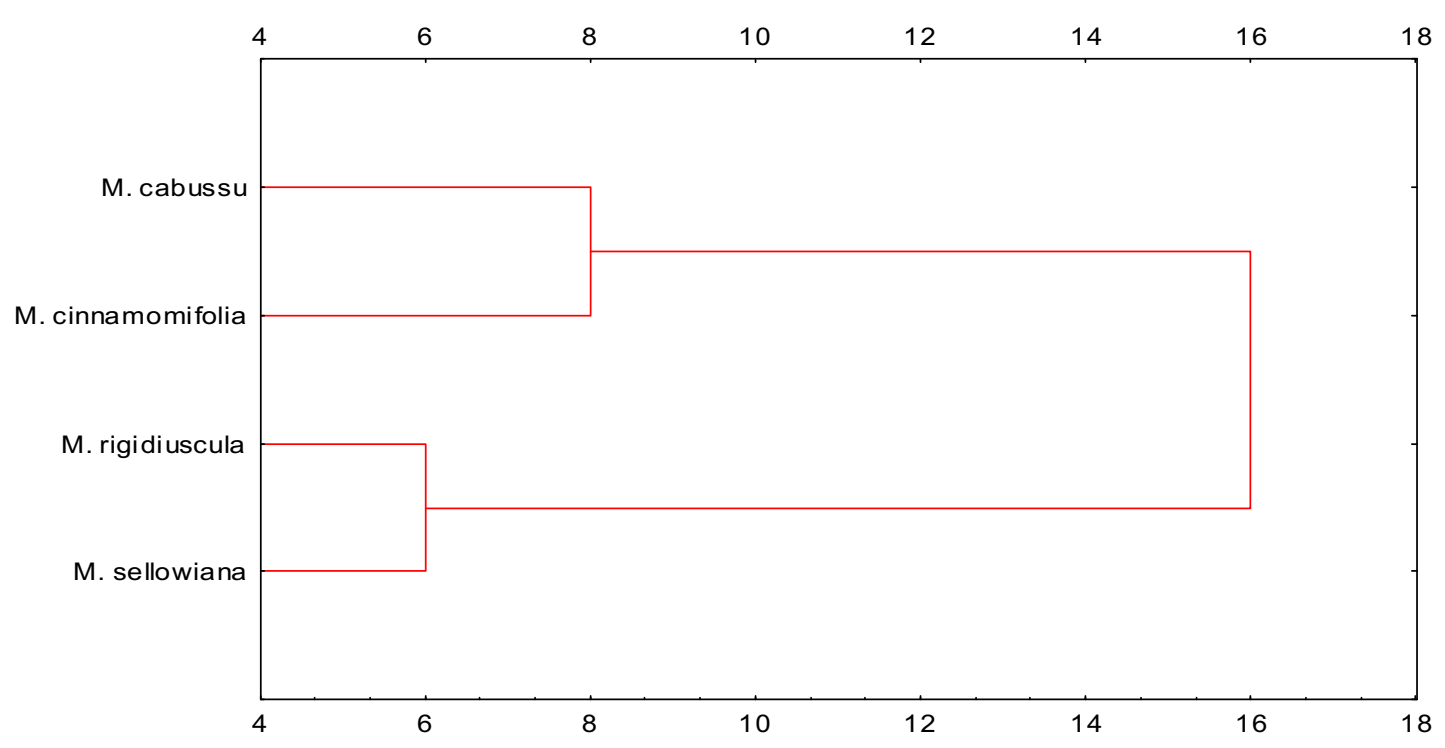

Figura 20. Dendrograma elaborado a partir da matriz de distância entre as 4 espécies estudadas. (Distância de Manhattan pelo método de peso proporcional - Weighted pair-group average).

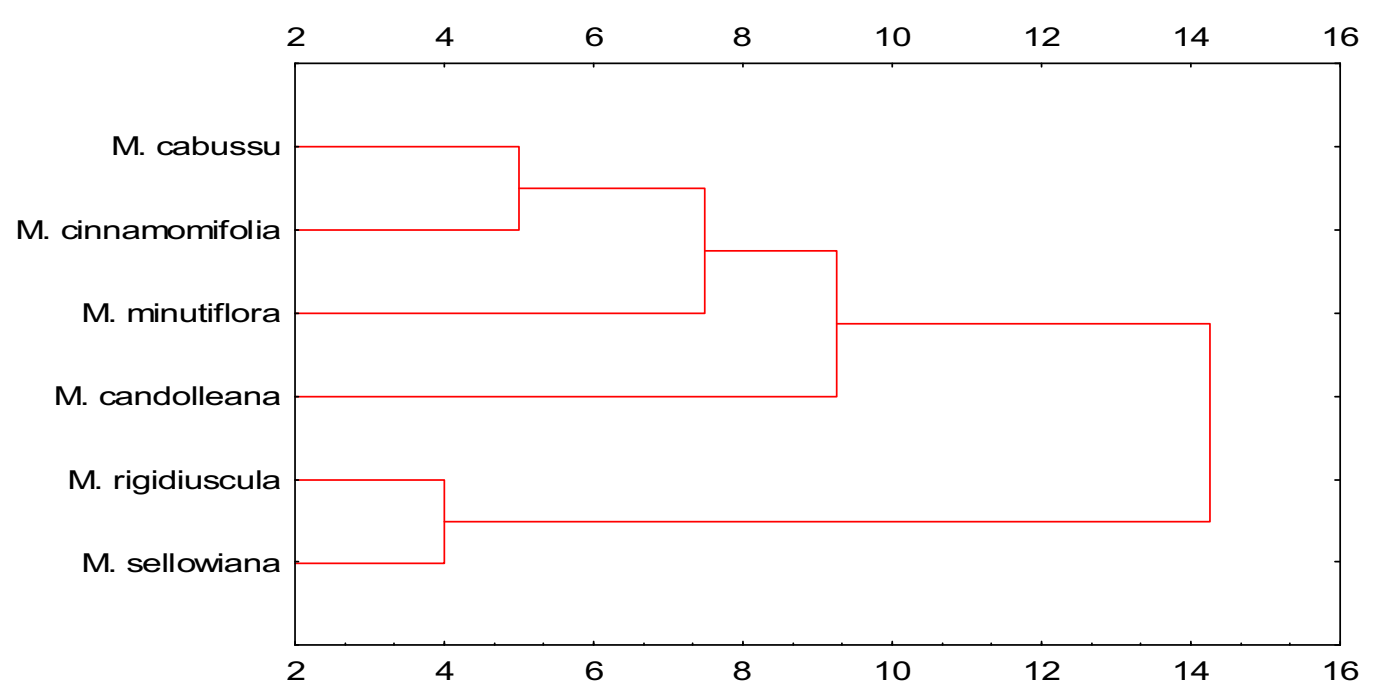

Figura 21. Dendrograma elaborado a partir da matriz de distância entre as 4 espécies estudadas e as 2 espécies descritas na literatura. (Distância de Manhattan pelo método de peso proporcional - Weighted pair-group average). 


\section{DISCUSSÃO E CONCLUSÕES}

As espécies estudadas se caracterizam pela presença de camadas de crescimento distintas; vasos solitários, em múltiplos e raramente em cachos, com arranjo difuso; placas de perfuração simples; pontoações intervasculares pequenas, alternas e ornamentadas; parênquima axial paratraqueal escasso; raios compostos de células predominantemente quadradas e eretas; fibras semelhantes a parênquima, fibras septadas e não septadas, gelatinosas e não gelatinosas com pontoações na parede radial. Tais características correspondem ao referido na literatura para a subfamília Melastomatoideae, tribo Miconieae e gênero Miconia (Van Vliet et al. 1981; Van Vliet \& Baas 1984; Welle \& Koek-Noorman 1981).

Faixas de fibras que se assemelham a parênquima são constantemente observadas na família Melastomataceae e no gênero Miconia. Hess (1946), em sua chave XIX para madeiras com fibras semelhantes a parênquima, observou que nas Melastomataceae essas fibras apresentam-se frouxamente agregadas em pequenas áreas ou em faixas irregulares com largos espaços intercelulares. Welle \& Koek-Noorman (1981) referem-se a um pseudoparênquima nas Melastomataceae neotropicais que consiste de faixas de fibras ou de fibras e células de parênquima em proporções variadas. As fibras que compõem essas faixas são muito similares às células fusiformes, podendo ser septadas ou não e diferem das fibras do tecido ao redor pelo espessamento da parede, diâmetro do lúmen e comprimento das células. Welle \& Koek-Noorman (1978) estudando alguns representantes do gênero Miconia, descreveram a presença de faixas tangenciais mais ou menos contínuas que consistem de fibras semelhantes a parênquima, séries de parênquima e células de parênquima fusiforme em quantidades variadas. As fibras que compõem essas faixas tangenciais diferem em diversos aspectos do tecido fibroso típico ao redor, elas possuem paredes mais delgadas, lúmen mais largo e em seção transversal apresentam contorno arredondado e espaços intercelulares. As fibras típicas, contudo, têm freqüentemente paredes gelatinosas e não apresentam espaços intercelulares. Ambos os tipos de fibras são quase sempre septados.

Nas quatro espécies analisadas neste estudo, observa-se que o arranjo em faixas contínuas das fibras semelhantes a parênquima separa Miconia cinnamomifolia de Miconia cabussu, Miconia rigidiuscula e Miconia sellowiana. Nesta última, essas fibras ocorrem em pequenas áreas ou estão associadas ao anel de crescimento, assemelhando-se a um parênquima marginal. Em Miconia cabussu e Miconia rigidiuscula elas ocorrem em pequenas áreas ou em faixas irregulares. Em todas as espécies os aspectos que diferenciam o tecido fibroso semelhante a parênquima do tecido fibroso típico são bastante semelhantes aos referidos acima para a família Melastomataceae e para o gênero Miconia. Em Miconia cabussu e M. sellowiana as fibras que se assemelham a parênquima apresentam as paredes mais delgadas e o lúmen mais largo do que as fibras típicas, além de poucos espaços intercelulares notados em seção transversal. Em M. rigidiuscula essas fibras diferem do tecido fibroso típico somente por terem lúmen mais largo e poucos espaços intercelulares. Já em M. cinnamomifolia elas apresentam paredes mais delgadas, lúmen mais largo e muitos espaços intercelulares. Com relação ao parênquima axial, em nenhuma das espécies estudadas está associado às faixas de fibras semelhantes a parênquima, sendo do tipo paratraqueal escasso. A escassez deste tecido de armazenamento é provavelmente compensado pelo desenvolvimento das fibras semelhantes a parênquima e das fibras septadas que irão funcionar como séries de parênquima (Record 1944; Van Vliet et al. 1981).

A forma e o tamanho das pontoações intervasculares, raio-vasculares e parênquimovasculares, assim como a largura e os tipos de células que compõem os raios são 
considerados por Welle \& Koek-Noorman (1981) de valor diagnóstico para os níveis de gênero ou espécie. Segundo estes autores, a associação entre a forma e o tamanho das pontoações intervasculares e a forma e o tamanho das pontoações raio e parênquimovasculares constituem também um dado importante para distinguir os três grupos de gêneros que compõem as Melastomataceae neotropicais. O gênero Miconia se enquadra no grupo 2 por possuir pontoações intervasculares circulares a ovais com tamanho entre 3-10 $\mu \mathrm{m}$, e pontoações raio e parênquimovasculares mais alongadas. Todas as espécies analisadas apresentaram pontoações intervasculares medindo entre $3-10 \mu \mathrm{m}$ e pontoações raio e parênquimo-vasculares alongadas, além de raios integrados por células em sua maioria quadradas e eretas. Pontoações raio-vasculares de dois diferentes tipos e tamanhos em uma mesma célula do raio separam Miconia rigidiuscula das outras três espécies. Pontoações intervasculares circulares e raios unisseriados distinguem Miconia cabussu e Miconia cinnamomifolia de Miconia rigidiuscula e Miconia sellowiana. Estas últimas apresentam pontoações intervasculares poligonais e raios bi e trisseriados.

Outras características, no entanto, separam as espécies aqui estudadas, são elas: demarcação das camadas de crescimento em Miconia cabussu e M. sellowiana; presença de traqueóides em Miconia cabussu; arranjo contínuo das fibras semelhantes a parênquima e comprimento dos elementos de vaso em $M$. cinnamomifolia; freqüência dos elementos de vaso em $M$. rigidiuscula e $M$. sellowiana. Dentre essas características, somente as qualitativas foram consideradas de valor diagnóstico, já que o comprimento e a freqüência dos elementos de vaso são comprovadamente determinados pelo ambiente e variam em uma mesma espécie sob condições ambientais diferentes (Metcalfe \& Chalk 1985).

Cogniaux (1887/1888) subordinou as espécies de Miconia a diferentes seções estabelecidas por ele com base em caracteres morfológicos. Miconia cabussu e $M$. cinnamomifolia foram subordinadas à seção Glossocentrum, já $M$. rigidiuscula e $M$. sellowiana foram subordinadas à seção Chaenanthera. Segundo Wurdack (1962) e Martins et al. (1996), M. sellowiana, no entanto, pode ser enquadrada à seção Glossocentrum pelo fato de suas anteras serem morfologicamente mais parecidas com a das espécies desta seção.

A análise anatômica do lenho das espécies aqui estudadas demonstrou existir uma tendência de agrupamento entre Miconia cabussu e $M$. cinnamomifolia e entre $M$. rigidiuscula e $M$. sellowiana, o que está de acordo com a classificação de Cogniaux (1887/ 1888). As características que promove este agrupamento são: vasos de diâmetro médio, pontoações intervasculares circulares e raios unisseriados muito finos em $M$. cabussu e $M$. cinnamomifolia; vasos de diâmetro pequeno, pontoações intervasculares poligonais e raios bi e trisseriados finos em $M$. rigidiuscula e M. sellowiana. Por outro lado, nenhuma característica de $M$. sellowiana assemelhase às características anatômicas das duas espécies da seção Glossocentrum aqui estudadas (M. cabussu e $M$. cinnamomifolia), o que não corresponde ao que foi observado por Wurdack (1962) e Martins et al. (1996) com respeito aos aspectos morfológicos.

No levantamento da literatura sobre anatomia de madeira do gênero Miconia não foi encontrada qualquer descrição das espécies pertencentes à seção Chaenanthera. Quanto à seção Glossocentrum, no entanto, foram encontradas duas espécies descritas: Miconia candolleana (Barbosa et al. 1977/78) e $M$. minutiflora (Welle \& Koek-Noorman 1981). Uma análise comparada feita com essas duas espécies e com as quatro espécies aqui estudadas evidenciou a formação de um primeiro grupo principal constituído por $M$. rigidiuscula e $M$. sellowiana. Miconia 
cabussu, M. candolleana, M. cinnamomifolia e M. minutiflora não formaram um grupo único, mas apresentaram uma tendência a agruparem-se entre si. Além disso, essas espécies se posicionaram a uma distância considerável daquele primeiro grupo formado, indicando uma semelhança com a classificação de Cogniaux (1887/1888). As características que diferenciam $M$. cabussu, M. candolleana, M. cinnamomifolia e $M$. minutiflora de $M$. rigidiuscula e $M$. sellowiana são novamente diâmetro dos vasos, forma das pontoações intervasculares e largura dos raios.

Deve-se levar em consideração, no entanto, que o diâmetro dos vasos é uma característica nitidamente determinada pelo ambiente (Metcalfe \& Chalk 1985), e, portanto, sujeita a modificações fenotípicas, fato que a torna menos útil para o agrupamento das espécies em questão. Já a forma das pontoações e a largura dos raios podem oferecer bom valor diagnóstico para este agrupamento.

\section{AGRADECIMENTOS}

Ao Instituto de Pesquisas Jardim Botânico do Rio de Janeiro e ao Programa Mata Atlântica. Ao pesquisador José Fernando Baumgratz pelas sugestões relacionadas ao tema. À Dra. Claudia Franca Barros pela revisão do trabalho. À Prof ${ }^{a}$. Cátia Henriques Callado pelas sugestões e ajuda na realização da análise estatística. Ao Prof ${ }^{\circ}$. Osnir Marquete pela ajuda prestada na confecção das pranchas. À tecnologista de madeira Inês Cosme Neves Grillo e ao técnico de laboratório Paulo Rogério Ferreira Dias pela confecção das fotomicrografias.

\section{REFERÊNCIAS BIBLIOGRÁFICAS}

Baas, P. 1981. A Note on Stomatal Types and Crystals in the leaves of Melastomataceae. Blumea 27: 475-479.

Barbosa, O.; Baitello, J.b.; Mainieri, C.; Montagna, R.g. \& Negreiros, O. C. de. 1977/78. Identificação e fenologia de espécies arbóreas da Serra da Cantareira (São Paulo). Silvicultura, $S$. Paulo 11/12: 1-168.

Barroso, G. M.; Peixoto, A. L.; Ichaso, C. L. F.; Costa, C. G.; Guimarães, E. F. \& Lima, H. C. de. 1984. Sistemática de Angiospermas do Brasil. Vol. II. Viçosa, UFV, Impr. Univ. 377p.

Baumgratz, J. F. A. 1980. Miconias do município do Rio de Janeiro. Seção Miconia DC. (Melastomataceae). Rodriguésia 32: 73-95. 1984. Miconias

do Estado do Rio de Janeiro. Seção Chaenanthera Naud. (Melastomataceae). Rodriguésia 36: 45-58.

Burger, L. M. \& Richter, H. G. 1991. Anatomia da Madeira. Nobel SP. $154 \mathrm{p}$.

Cogniaux, A. 1887/1888. Miconia, Melastomataceae. In: Martius \& $A G$. Eichler, Flora Brasiliensis. Vol. 14 (4). Frid. Fleischer, Lipsiae. 1891. Mélastomatacées. In: Monographie Phanerogamarum. VII (eds. A. \& C. de Candolle). Masson. Paris.

COPANT. 1973. Comision Panamericana de Normas Técnicas Pan American Standards Commission. Esquema $1^{\circ}$ de Recomendação.

Dahlgren, R. \& Thorne, R. F. 1984. The Order Myrtales: Circumscription, Variation, and Relationships. Ann. Missouri Bot. Gard. 71: 633-699.

Dop, D. \& Gautié, A. 1909. Manuel de Technique Botanique. Paris, F. R. de Sudeval, Ed. XXXII. 594p.

Fedalto, L. C. 1982. Estudo anatômico do lenho de Bixa arborea Huber. Acta Amazonica 12 (2): 389-399.

Gonzales - Rodriguez, E.; Maestrini, S. Y.; Valentin, J. L. \& Rivera - Tenenbaum, D. 1989. Variação da composição específica do fitoplâncton de Arraial do Cabo (RJ, Brasil) após enriquecimento 
com nutrientes..Nerítica, Pontal do Sul, PR, 4 (1/2): 33-56.

Guedes-Bruni, R. R. \& Lima, M. P. 1994. Abordagem geográfica, fitofisionômica, florística e taxonômica da Reserva Ecológica de Macaé de Cima. In: Lima, M. P. \& Guedes-Bruni, R. R. 1994. Reserva Ecológica de Macaé de Cima. Nova Friburgo - RJ. Aspectos florísticos das Espécies Vasculares. Vol. I. 404p.

Hess, R. W. 1946. Keys to american woods (continued) ${ }^{1}$. Trop. Woods 85: 11-19.

IAWA Committee. 1989. IAWA list of microscopic feature of hardwood identification. IAWA Bull. 10 (3): 219332.

Jane, F. W. 1956. The structure of wood. Londres Adam \& Black. 480p.

Johansen, D. A. 1940. Plant microtechnique. New York, Mc.Graw - Hill Book Company, XI. 523p.

Johnson, L. A. S. \& Briggs, B. G. 1984. Myrtales and Myrtaceae-A Phylogenetic Analysis. Ann. Missouri Bot. Gard. 71: 700-756.

Keating, R. C. 1984. Leaf Histology and its Contribution to Relationships in the Myrtales. Ann. Missouri Bot. Gard. 71: 801-823.

Martins, A. B.; Semir, J.; Goldenberg, R. \& Martins, E. 1996. O gênero Miconia Ruiz \& Pav. (Melastomataceae) no Estado de São Paulo. Acta Bot. Bras. 10 (2): 267-315.

Mentink, H. \& Baas, P. 1992. Leaf Anatomy of the Melastomataceae, Memecylaceae, and Crypteroniaceae. Blumea 37: 189-225.

Metcalfe, C. R. \& Chalk, L. 1985. Anatomy of the Dicotyledons. Vol. II. Wood structure and conclusion of the general introduction. 2nd. ed. Clarendon Press - Oxford. 297p + il.

Programa Mata Atlântica. 1993. Relatório final. Jardim Botânico do Rio de Janeiro/IBAMA 54p + anexos.
Record, S. J. 1944. Keys to american woods. Trop. Woods 78: 35-45. \& Mell, C. D. 1924. Timbers of Tropical America. New Haven: Yale Univers. Press. 610p.

Relatório Técnico do Programa Mata Atlântica do Jardim Botânico do Rio de Janeiro. 1995. JBRJ/Ibama/Fundação Botânica Margaret Mee/CNPq. 56p + anexos.

Renner, S. S. 1993. Phylogeny and classification of the Melastomataceae and Memecylaceae. Nord. J. Bot. 13: 519-540.

Sass, J. E. 1958. Elements of botanical microtechnique. New York - London, Mc.Graw - Hill Book Company Inc. 222p.

Van Vliet, G. J. C. M. 1981. Wood anatomy of the paleotropical Melastomataceae. Blumea 27: 395-462.

. \& Baas, P. 1984. Wood anatomy and classification of the Myrtales. Ann. Missouri Bot. Gard. 71: 783-800.

; Koek-noorman, J. \& Welle, B. J. H. Ter. 1981. Wood anatomy, classification and phylogeny of the Melastomataceae. Blumea 27: 463473.

Welle, B. J. H. Ter \& Koek-noorman, J. 1978. On fibres, parenchyma and intermediate forms in the genus Miconia (Melastomataceae). Acta Bot. Neerl. 27: 1-9.

1981. Wood anatomy of the neotropical Melastomataceae. Blumea 27: 335394.

Wurdack, J. J. 1962. Melastomataceae of Santa Catarina. Sellowia 14: 109-217. 